
$\$$ Research Square
Preprints are preliminary reports that have not undergone peer review.
They should not be considered conclusive, used to inform clinical practice, or referenced by the media as validated information.

\title{
Risk score to predict time-to sputum smear and culture conversions in patients treated with shorter MDR-tuberculosis regime in Guinea (Conakry): A retrospective cohort study.
}

\author{
Alhassane Diallo \\ INSERM, U1137-IAME, CIC EC 1425, University Paris Diderot \\ Boubacar Djelo Diallo ( $\sim$ diallodjelo@yahoo.fr) \\ Universite Gamal Abdel Nasser de Conakry https://orcid.org/0000-0002-3015-6722 \\ Lansana Mady Camara \\ Universite Gamal Abdel Nasser de Conakry Faculte de Medecine Pharmacie et Odontostomatologie \\ Gladys Djuiga Fotso \\ Universite Gamal Abdel Nasser de Conakry Faculte de Medecine Pharmacie et Odontostomatologie

\section{Souleymane Camara}

Centre antituberculeux de la carrière, Conakry

\section{Boubacar Bah}

Centre antituberculeux de la carrière, Conakry

\section{Magassouba Aboubacar Sidiki}

Programme national lutte antituberculeux

\section{Alpha Oumar Barry}

Service Pneumo-Phtisiologie CHU Ignace Deen

\section{Thierno Hassane Diallo}

Service de Pneumo-Phtisiologie CHU Ignace Deen

\section{Camara Aboubacar}

Service pneumo-phtisiologie CHU Ignace Deen

Néné Ramata Bah

Laboratoire national des mycobactéries, Conakry

Fulgence N'Zabintawali

Laboratoire national des mycobactéries, Conakry

Souleymane Hassane Haroune

Fondation Damien Conakry

Miguel Lotshutshu

Urgences réanimation centre hospitalier Sud Essonnes CHSE, Paris

Mamadou Hassimiou Diallo

Centre Population et Developpement, Paris Descartes 


\section{Oumou Younoussa Sow}

Universite Gamal Abdel Nasser de Conakry Faculte de Medecine Pharmacie et Odontostomatologie

\section{Research article}

Keywords: Conversion, Multidrug-resistant, Tuberculosis, Guinea

Posted Date: September 18th, 2019

DOl: https://doi.org/10.21203/rs.2.14492/v1

License: (c) (1) This work is licensed under a Creative Commons Attribution 4.0 International License. Read Full License

Version of Record: A version of this preprint was published at Central African Journal of Public Health on January 1st, 2020. See the published version at https://doi.org/10.11648/j.cajph.20200601.15. 


\section{Abstract}

Background Sputum smear and culture examination were used as bacteriological marker to monitor multidrug-resistant tuberculosis (MDR-TB) treatment. We aimed to identify the strongest contributing factors that affect time-to sputum bacteriological conversion, and to develop a practical risk score

Methods Patients treated with shorter MDR-TB treatment regime between June 07, 2016 and June 22, 2018 from three major drug-resistance TB centres in Guinea, who had a positive smear or culture at baseline, and who had rifampicin resistance were analysed. Sputum bacteriological conversion was defined as two consecutive negative smear or culture taken at least 30 days apart. Cox regression model was used to analyse time-to initial sputum conversion, and to identify influencing factors. A simple risk score was constructed using the regression coefficients from each final model. Time-dependent AUC was used to determine the optimal time points of bacteriological conversion.

Results $75 \%$ (173/232) of patients with a positive smear and culture at baseline were analyzed. A total, $90.2 \%(156 / 173)$ and $89 \%(154 / 173)$ of the patients had smear and culture conversions in a median of 59 days (inter-quartile range: $59-61)$ respectively. Lower colony count $(<3+)$ of initial culture $(\mathrm{aHR}=$ $1.52,1.09-2.14)$ and previously history of TB treatment $(\mathrm{aHR}=2.10,1.26-3.51)$ were more likely to have culture conversion, while only the higher $\mathrm{BMI}(\mathrm{aHR}=1.10,1.03-1.18)$ was associated with rapid smear conversion. The predicted risk score from independent predictors showed good discrimination $(0.855 \pm 0.023$ and $0.883 \pm 0.02$ respectively for smear and culture conversions). Time-to initial culture conversion provided a better discriminative capacity (AUC) to detect patients with higher chance to being treatment success during the first three months.

Conclusion The identified predictors can be considered to improve the management of MDR-TB patients. The optimal time points for culture conversion was three months in shorter regime. Keywords: Conversion, Multidrug-resistant, Tuberculosis, Guinea.

\section{Background}

Multidrug-resistant (MDR)-TB defined as resistance at least to isoniazid (INH) and rifampicin (R) is a major public health problem with an estimated 600000 new cases reported by the World Health Organization (WHO) in 2016 [1].

Regardless the MDR-TB treatment regime (12 or 24 months), the WHO recommend the using sputum smear or culture conversion as a proxy marker for a successful MRD-TB treatment outcome [1]. Recently, many efforts have been made to validate these MDR-TB treatment efficacy markers including the optimal time of measurement and the influencing factors [2-10]. One of these studies concluded that the validity of culture conversion is significantly higher than sputum smear conversion, and the four months schedule is a common optimal time for culture and smear conversion [10]. Nevertheless, the influencing factors of time-to initial sputum smear and culture conversions varied from one study to another [2-5]. 
Moreover, for MDR-TB patients who treated by the shorter regime of 9 months, little data are available about the optimal time-to sputum smear or culture conversion, as well as the factors influencing time to sputum bacteriological conversion. Here, we reported data from a retrospective cohort study of patient treated with a standardized shorted treatment regimen of 9-months in Guinea. We aimed to identify the strongest contributing factors that affect time to sputum smear and culture conversions, and to develop a practical prognostic risk score.

\section{Methods}

\section{Study design and population}

We conducted a retrospective multicenter longitudinal cohort study from three major drug-resistance TB centres in Guinea (Ignace-Deen, Carrière and Tombolia). In this study, data for patients aged 18 years with positive sputum smear and culture at baseline, and confirmed MDR-TB who started a shorter MDR-TB treatment regime between June 07, 2016 and June 22, 2018 were analysed. These patients were seen at baseline and followed by monthly visit for 9 months. Both sputum smear microscopy tests and sputum mycobacterial cultures were performed monthly in these centres until the end of therapy. Patients who were younger than 18 years and patients that tested sputum smear and culture negative at baseline were excluded from the analysis.

\section{Bacteriology, drug susceptibility testing, and treatment regimens for MDR-TB in Guinea,}

The diagnosis of drug-resistance was done by the Xpert MTB/RIF test. For others anti-TB drugs, the drug susceptibility testing (DST), based on solid culture (Lowenstein-Jensen) were done late, and in a very partial way. Second-line anti-TB drugs were not routinely tested. According to the guideline for the MDR management in Guinea [11], naive patients for second-line anti-TB drugs were treated with a short 9month regimen, consisting of an intensive phase lasting a minimum of 4 months including moxifloxacin, kanamycin, clofazimine, prothionamide, pyrazinamide, ethambutol, and INH at high dose. The intensive phase was then followed by the continuation phase during 5 months and consisting of administration of four drugs: moxifloxacin, clofazimine, pyrazinamide, and ethambutol. Sputum smears and cultures were obtained monthly during the MDR-TB treatment duration.

\section{Data collection}

Data was collected using a case report form (CRF) from the MDR-TB registry that containing sociodemographic, clinical data, and the laboratory test results (sputum smear or culture measurements) for all patients who were admitted in the three major TB centres outpatient care. Additional information 
was completed with data from the patients' clinical files. The following clinical and demographic data record were extracted: age, gender, residence, comorbidity, HIV status, history of the previously treated TB, the presence of cavities on chest X-ray determined by the senior radiologist, baseline data on weight, clinical symptoms (as chest pain or cough), biological data, and baseline as well as repeated measurements of sputum smear and culture during treatment course. Additionally, we extracted adherence to MDR-TB treatment status during follow-up based on the proportion of days covered (PDC). For each patient, we calculated a PDC by dividing the number of days covered with MDR-TB treatment delivered over one month by 31. Then, we considered a minimal value of PDC during follow-up for each patient as a marker of adherence to MDR-TB treatment. Conventionally, the PDC was dichotomized between good adherence if the PDC was 0.8 or more and poor adherence otherwise [12].

\section{Outcome definitions}

Sputum smear conversion was defined as two consecutive negative sputum smears taken at least 30 days apart following an initial positive sputum smear. Similarly, culture conversion was defined as two consecutive negative culture taken at least 30 days apart following an initial positive sputum culture. Time-to initial sputum smear (or culture) conversion was defined as the time in months from the date of start of MDR-TB treatment to the date of specimen collection for the first of two consecutive negative sputum smears (or culture) results, even if a subsequent positive sputum smears (or culture) result occurred later. Smear and culture reversions to positive were defined when at least one subsequent positive smear or culture was recorded after the initial conversion. Time-to smear (or culture) reversion was defined as the same manner for time-to initial sputum smear (or culture) conversion. According to the revised WHO recommendations in 2013 [13], we classified the treatment outcomes into two categories (successful or unsuccessful). Successful treatment outcomes corresponded to patient who declared as either "cured" or "treatment completed". Unsuccessful treatment outcomes included "treatment failure", or "death form any reason" or "lost to follow-up" or "not evaluated".

\section{Statistical analysis}

Means (standard deviation; SD) or frequencies (percentage) were used to describe continuous and categorical variables at baseline. Time-to initial sputum smear and culture conversions every month was estimated using the Kaplan-Meier method and differences in survival time were compared with a log-rank test. Data for patients who did not achieve sputum smear (or culture) conversion were censored before the last follow-up. Univariate Cox regression was used to identify prognostic factors associated with timeto initial sputum smear (or culture) conversion, and then candidates with a p-value less than 0.10 were entered into the multivariate Cox regression. The independent predictors of time-to initial sputum smear (or culture) conversion from multivariate regression were selected through a backward procedure based on the lowest Akaike information criterion. Hazard ratio (HR) together with their $95 \%$ confidence interval (Cl) were used as association measures. Assumption of hazard proportionality and log-linearity were verified. 
From each final Cox model, we computed a clinical raw prognostic score (linear predictor) by summing the contribution of each individual factor, based on the estimate for each factor in the model. To derive the optimal cut-off point score, the receiver-operating characteristic (ROC) applying the Youden's index method was used [14]. Performance measures including the sensitivity, specificity, and positive and negative predictive values (PV) were estimated at this optimal threshold. Patients were then divided into two clinical risk score groups according the optimal threshold: "low chance to conversion" and "high chance to conversion".. Finally, the time-dependent area under curve (AUC) as well as their $95 \%$ confidence interval $(\mathrm{Cl})$ of the difference in AUC between smear and culture conversions were used to determine the optimal time points of sputum smear and culture conversions. The final model was internally validated using the 1,000 samples bootstrap procedure. All data analyses were done in $\mathrm{R}$ (version 3.5.1). Significance was defined as a p-value less than 0.05 , and all tests were two-sided.

\section{Results}

\section{Socio-demographic and clinical characteristics at baseline}

A total of 232 patients with MDR-TB were diagnosed and followed during treatment period, among them

173 patients (75\%) who meet the inclusion criterion were analyzed. The clinical characteristics at baseline between exclude and analysed patients were comparable (Appendix, Table S1) except for gender $(p=0.0383)$, HIV status $(p=0.0266)$, years of inclusion $(p=0.0194)$, leucocyte $(p=0.0025)$ and lymphocyte counts $(p<0.0001)$. The mean age of patients was 34.011 .3 years, with the majority of them were male (72\%), leaving in urban area (77\%), with a mean BMI of $17.82 .8 \mathrm{~kg} / \mathrm{m}^{2}$. Most patients presented cough (94\%) or night sweat (53\%), 34 (20\%) patients were HIV positive, and 13 (9\%) patients had cavitary lesions on initial chest X-ray. According to the history of TB, 146 (85\%) patients were previously treated, $49(28 \%)$ and $85(49 \%)$ had a colony count of initial sputum smear and culture higher than $3+$ respectively (Table 1 ). Among those patients who converted their sputum smear and culture, $95 \%$ and $93 \%$ had good adherence to the MDR-TB treatment. Overall, $77.5 \%(134 / 173)$ of patients were cured or completed their treatment.

\section{Sputum smear status and conversion time}

Among 173 patients with sputum smear positive at baseline, $17(9.8 \%)$ patients failed to converted their sputum smear to negative during treatment period (Figure 2A), while 156 (90.2\%) patients had smear conversion in a median of 59 days (inter-quartile range: 59-61). Patients who did not convert died more frequently (57\%), and were more likely to have lower BMI $\left(16.42 .4 \mathrm{~kg} / \mathrm{m}^{2}\right)$. Figure 1 showed a decline number of patients who converted over time. According to the MDR-TB treatment outcomes, we found that the median time-to smear conversion was comparable between patients with successful (59 days, $95 \% \mathrm{Cl}, 31-61)$ and poor treatment outcomes (61 days, 95\% Cl, 59-92, $\mathrm{p}=0.0684$ ) (Figure 2B). 


\section{Sputum culture status and conversion time}

154 (89\%) patients converted their culture in a median of 59 days (inter-quartile range: $59-61$ ), and 19 (11\%) patients did not have culture conversion (Figure 2C). As the sputum smear, the number of patients who converted their culture decrease over time (Figure 1), and patients who did not convert their culture died more frequently (57\%), and were more likely to have lower BMI $\left(16.42 .4 \mathrm{~kg} / \mathrm{m}^{2}\right)$. Contrariwise, the median time-to culture conversion among patients with successful MDR-TB treatment was significantly shorted at 31 days $(95 \% \mathrm{Cl}, 31-59)$ compared to patients with poor MDR-TB treatment outcomes, 89 days $(95 \% \mathrm{Cl}, 61-92, \mathrm{p}=0.0008)$ (Figure 2D).

\section{Sputum smear and culture status reversion time}

The number of patients who had at least one positive smear result (i.e. smear reversion) after an initial smear conversion was $9(5.2 \%)$. The median time of smear reversion from date of MDR-TB treatment initiation was 212 days (IQR: 181-275 days). The corresponding number of patients with culture reversion was 11 (6.4\%), with a median time of culture reversion from date of MDR-TB treatment initiation of 150 days (IQR: $97-255$ days).

\section{Predictors of time-to initial sputum smear and culture conversion}

In univariate Cox regression (Appendix, Table S1), predictor associated with shorter time-to initial sputum smear conversion was the higher $\mathrm{BMI}(\mathrm{HR}=1.09,95 \% \mathrm{Cl}, 1.02-1.17 ; \mathrm{p}=0.0089)$. The corresponding predictors for shorter time-to initial culture conversion were the previously history of TB treatment $(\mathrm{HR}=$ $1.68,1.03-2.74 ; p=0.0384)$, lower colony count $(<3+)$ of initial culture $(H R=1.42,1.02-1.98 ; p=$ $0.0359)$, and successful of MDR-TB treatment $(H R=1.92,1.20-3.07 ; p=0.0068)$. Independently to the MDR-TB treatment outcomes, the only strongest contributing factor from multivariate Cox model for the shorter time-to initial sputum smear conversion (Table 2) was the higher BMI (adjusted hazard ratio (aHR) $=1.10,1.03-1.18 ; p=0.0048)$. The corresponding strongest contributing factors for shorter time-to initial culture conversion (Table 2 ) were the previously history of TB treatment (aHR $=2.10,1.26-3.51 ; \mathrm{p}=$ $0.0046)$, and lower colony count $(<3+)$ of initial culture $(a H R=1.52,1.09-2.14 ; p=0.0148)$. In the internal validation, the uncertainties measured by the bootstrapping procedure were close to the estimated HR (Table 2), suggesting robustness of the final models.

\section{Clinical risk score of the time-to initial sputum smear and culture conversion}

From each final model (time-to initial sputum smear and culture conversions), we derived a clinical risk score and we did an optimal threshold search. For the time-to initial culture conversion, 0.08-point score was found to be optimal, where $68 \%$ of patients belong to the high chance conversion group and $32 \%$ of patients classified to low chance conversion group. Patients in the higher group had 2.29 times $(\mathrm{HR}=$ 
2.29 [95\% Cl, 1.55-3.39]; $\mathrm{p}<0.0001$ ) chance to convert their culture. In addition, the median time-to the culture conversion was significantly shorter among these patients (high chance conversion group, 31 days, $95 \% \mathrm{Cl}, 31-59)$ compared to those patients with low chance conversion, 52 days $(95 \% \mathrm{Cl}, 61-90$, $\mathrm{p}<0.0001$ ) (Figure 3B). The estimated performance measures based on this optimal threshold for predicting time-to initial culture conversion were $77 \%$ for sensitivity, $100 \%$ for specificity, $100 \%$ for positive predictive values, and $36 \%$ for negative predictive values. The corresponding optimal threshold for the time-to initial sputum smear conversion was -0.14 , where $78 \%$ and $22 \%$ of patients belong to the high and low chance conversion groups respectively. The median time-to the smear conversion was significantly shorter among patients belonging in the higher chance group, 59 days ( $95 \% \mathrm{Cl}, 31-61$ ) compared to those patients with low chance smear conversion, 90 days $(95 \% \mathrm{Cl}, 61-92, \mathrm{p}=0.0006)$ (Figure 3A), with an associated HR of 2.00 [95\% Cl, 1.35-2.96; $p=0.0005]$. The corresponding estimated performance measures based on the optimal threshold for predicting time-to initial smear conversion were $77 \%$ for sensitivity, $94 \%$ for specificity, $99 \%$ for positive predictive values, and $32 \%$ for negative predictive values. The overall discriminative capacity ( $A U C \mathrm{SE}_{\mathrm{AUC}}$ ) of the risk score to predict time-to initial smear and culture conversions were 0.8550 .023 and 0.8830 .025 respectively. Figure 4 showed the comparison of the predictive power of each risk score to detect patients with higher chance to achieve treatment success during the first three months. Although the difference was not statistically significant, the time-to initial culture conversion seemed to provide a better discriminative capacity (time-dependent AUC) to detect patients with higher chance to being treatment success during the first three months. While the discriminative capacity of time-to initial culture conversion increased over time to reach 0.88 at three months, the time-dependent AUC for time-to initial sputum smear conversion decreased. In addition, time-dependent positive and negative predictive values of culture conversion were higher than smear conversion ( $6 \%$ vs $5 \%$ at one month, $68 \%$ vs $58 \%$ at two months, and $91 \%$ vs $84 \%$ at three months for positive predictive values, and $98 \%$ vs $97 \%$ at one month, $66 \%$ vs $66 \%$ at two months, and $55 \%$ vs $41 \%$ at three months for negative predictive values).

\section{Discussion}

To our knowledge, this is the first study to address the development and internally validation of risk score to the shorter sputum smear and culture conversions among patients with MDR-TB. In addition, this is the first study to address the development of clinical risk score to predict time-to sputum smear and culture conversions.

At the end of treatment, $89 \%$ of our patients converted their culture, which was comparable to the conversion rates reported in Ethiopia (88.6\%) [3], South Africa (89\%) [15], USA (85\%) [4], South Korea (91.7\%) [7], and Peru (92\%) [9], but higher than those reported in China (76.3\%) [5], and Indonesia (81\%) [8]. Nevertheless, the median culture conversion observed in our sample was comparable to those reported in the literature, ranging from 30 days to 91 days $[2,4,5]$.

In parallel, $90.2 \%$ of our patients achieved to sputum smear conversion in a median of 59 days. This median time to conversion was comparable to those previously reported in South Korea (56 days) [7], 
Peru (59 days) [9] and Dominican Republic (60 days) [16], but less days than in USA (93 days) [4], China (91.5 days) [5] and Ethiopia (72 days) [3]. Possible reasons for these discrepancies are selection bias, differences in MDR-TB regimens, genetic background, and differences between health-care systems.

The role of culture conversion as a biomarker of treatment success has been overlooked in many studies $[10,17]$. Our finding agreed with the literature report that patients who achieved earlier culture conversion were more likely to have successful MDR-TB treatment. Although, the higher rates of culture conversion in the present study are encouraging, additional efforts should be made to increase the rate of successful MDR-TB treatment up to $80 \%$ as recommended by the WHO.

Independently to the treatment outcomes, we found that the lower colony count of initial culture and previously treatment for TB were associated with more rapid culture conversion. While similar result had been found that the colony count of initial culture is predictive to the culture conversion [2], the majority of studies reported the colony count of initial sputum smear as predictor of culture conversion instead of colony count of initial culture [4,5]. In this study, the history of TB treatment was found to be associated to more rapid culture conversion, unlike to the study conducted by Holtz et al [2]. This association may be explained by the interaction between HIV infection and the metabolism of TB drugs [18]. Many literature data have been shown that in patients who infected by HIV infection, the absorption rate of MDR-TB drugs is lower compared to those without HIV infection $[19,20]$. In fact, among the 146 patients who were previously treated for TB, $14 \%$ are HIV positive compared to the 26 new cases patients in whom $54 \%$ are HIV positive.

Only the higher BMI was associated with a rapid smear conversion, after adjusted to the MDR-TB treatment outcomes. The impact of underweight on MDR-TB treatment outcomes is well established, and possible reasons are malnutrition, lower immunity or poor absorption from gastrointestinal tract $[7,8,21]$.

We have developed a clinical prognostic risk score for both time-to initial sputum smear and culture conversions. Risk scores had a good discriminative capacity and the identified thresholds optimized well the separation of the Kaplan Meier curves. Using the risk scores, we would be able to compare the predictive power of each biomarker (time-to initial sputum smear and culture conversions) to detect patients with higher chance to achieve treatment success during Treatment course. Because few patients converted their sputum smear or culture after three months, the time-dependent AUC were estimated only at the first three times visits after the initiation of MDR-TB treatment. Despite the absence of significance probably due to lack of power, we found that the predictive power of culture was higher than sputum smear, and the best discriminative capacity was reached at three months. Moreover, the time-dependent positive and negative predictive values for culture conversion were higher than sputum smear conversion. For example, the positive predictive value at three months of $91 \%$ indicate that patients who converted their culture at three months will have a $91 \%$ of chance to achieve successful MDR-TB treatment. These finding suggest that the culture is more sensitive to detect patients with higher probability of treatment success than sputum smear, and the optimal time point is three months. Similar results were found in Ethiopia that the validity of culture conversion is significantly higher than smear conversion in predicting 
MDR-TB treatment outcomes, and the optimum times for culture and smear conversion together is four months [10]. The slight difference concerning the optimal time point may be explained by the differences in MDR-TB regimens and the statistical models used to handle data. Nevertheless, future prospective cohort studies will be need to decide on the optimal time point of sputum smear and culture measurement.

Despite the higher discriminative capacity of our prognostic risk score in predicting smear and culture conversions, we need to externally validated it on independent samples including non-Guinean patients to establish her generalizability. Retrospective design, and some missing parameters such as diabetes status, smoking and alcohol use, and biomarkers were limits of this study, they might be further used to improve prognostic risk score.

\section{Conclusions}

In this study, we have developed and internally validated a clinical prognostic risk score, which has a higher predictive power to detect patients with great chance to sputum smear and culture conversions. Time-to initial culture conversion is the best marker to predict successful MDR-TB treatment, with an optimal time points of three months for patients treated with a shorter standardized regime. Our finding supports the WHO recommendation of adding culture to sputum smear in the better prediction of successful MDR-TB management.

\section{Declarations}

\section{Abbreviations}

BMI: body mass index; Cl: confidence interval; CRF: case report form; DST: drug susceptibility testing; HIV: human immunodeficiency virus; INH: isoniazid; MDR-TB: Multidrug-resistant tuberculosis; PV: predictive value; HR: hazard ratio; ROC: receiver-operating characteristic; SD: standard deviation; SE: standard error; TB: tuberculosis; WHO: World Health Organization.

\section{Ethics approval and consent to participate}

The study was approved by the National Ethics Committee for Heath Research (NECHR) attached to the Ministry of Health (Conakry, Guinea). The study was based in accordance to the Declaration of Helsinki, and the confidentiality of the data was guaranteed.

\section{Consent for publication}

Not applicable 


\section{Availability of data and materials}

The data is available upon authors request.

\section{Competing interests}

The authors declare that they have no competing interest in relation with this work.

\section{Funding}

None

\section{Author's contributions}

$A D$ contributed to the conception, organization the research project, conceived the study design, analysed the data, and drafted the manuscript, BDD contributed to the conception, organization the research project, supervision data collection, and critical revision to manuscript, GDF collected data and commented the manuscript, SC, LMC, OYS, contributed to the conception, organization the research project, and commented the manuscript, BB, MAS, AOB, THD, CA, NRB, FN, SHH, LM, and MAD commented the manuscript. All authors approved the final version of the manuscript.

\section{Acknowledgements}

We thank the National Tuberculosis Control Program for their collaboration, and Action Damien Foundation for their patients support during treatment.

\section{References}

1. World Health Organization: Global tuberculosis report 2017. Geneva, Swizerland: WHO press, 2017.

2. Holtz TH, Sternberg M, Kammerer S, Laserson KF, Riekstina V, Zarovska E, et al. Time to Sputum Culture Conversion in Multidrug-Resistant Tuberculosis: Predictors and Relationship to Treatment Outcome. Ann Intern Med. 2006;144(9):650.

3. Shibabaw A, Gelaw B, Wang S-H, Tessema B. Time to sputum smear and culture conversions in multidrug resistant tuberculosis at University of Gondar Hospital, Northwest Ethiopia. Cardona P-J, editor. PLOS ONE. 2018;13(6):e0198080.

4. Kurbatova EV, Gammino VM, Bayona J, Becerra MC, Danilovitz M, Falzon D, et al. Predictors of sputum culture conversion among patients treated for multidrug-resistant tuberculosis. Int $\mathrm{J}$ Tuberc Lung Dis. 2012;16(10):1335-43. 
5. Liu Q, Lu P, Martinez L, Yang H, Lu W, Ding X, et al. Factors affecting time to sputum culture conversion and treatment outcome of patients with multidrug-resistant tuberculosis in China. BMC Infect Dis 2018;18(1).

6. Lv L, Li T, Xu K, Shi P, He B, Kong W, et al. Sputum bacteriology conversion and treatment outcome of patients with multidrug-resistant tuberculosis. Infect Drug Resist. 2018;11:147-54.

7. Park H-O, Kim S-H, Moon S-H, Byun J-H, Kim J-W, Lee C-E, et al. Association between Body Mass Index and Sputum Culture Conversion among South Korean Patients with Multidrug Resistant Tuberculosis in a Tuberculosis Referral Hospital. Infect Chemother. 2016;48(4):317.

8. Putri FA, Burhan E, Nawas A, Soepandi PZ, Sutoyo DK, Agustin H, et al. Body mass index predictive of sputum culture conversion among MDR-TB patients in Indonesia. Int J Tuberc Lung Dis. 2014;18(5):564-70.

9. Tierney DB, Franke MF, Becerra MC, Alcántara Virú FA, Bonilla CA, Sánchez E, et al. Time to Culture Conversion and Regimen Composition in Multidrug-Resistant Tuberculosis Treatment. Pai M, editor. PLOS ONE. 2014;9(9):e108035.

10. Alene KA, Viney K, Yi H, McBryde ES, Yang K, Bai L, et al. Comparison of the validity of smear and culture conversion as a prognostic marker of treatment outcome in patients with multidrug-resistant tuberculosis. Hasnain SE, editor. PLOS ONE. 2018;13(5):e0197880.

11. National Tuberculosis Control Program Guinea: Annual report of TB control activity, 2018.

12. Hansen RA, Kim MM, Song L, Tu W, Wu J, Murray MD. Adherence: Comparison of Methods to Assess Medication Adherence and Classify Nonadherence. Ann Pharmacother. 2009;43(3):413-22.

13. WHO: Definitions and reporting framework for tuberculosis-2013 revision (updated 2014). Geneva: Switzerland World Health Organization; 2013.

14. Youden WJ. Index for rating diagnostic tests. Cancer. 1950;3(1):32-5.

15. Brust JCM, Lygizos M, Chaiyachati K, Scott M, van der Merwe TL, Moll AP, et al. Culture Conversion Among HIV Co-Infected Multidrug-Resistant Tuberculosis Patients in Tugela Ferry, South Africa. Pai M, editor. PLoS ONE. 2011;6(1):e15841.

16. Rodriguez M, Monedero I, Caminero JA, Encarnación M, Dominguez Y, Acosta I, et al. Successful management of multidrug-resistant tuberculosis under programme conditions in the Dominican Republic. Int J Tuberc Lung Dis. 2013;17(4):520-5.

17. Kurbatova EV, Cegielski JP, Lienhardt C, Akksilp R, Bayona J, Becerra MC, et al. Sputum culture conversion as a prognostic marker for end-of-treatment outcome in patients with multidrug-resistant tuberculosis: a secondary analysis of data from two observational cohort studies. Lancet Respir Med. 2015;3(3):201-9.

18. Saleri N, Dembele SM, Villani P, Carvalho ACC, Cusato M, Bonkoungou V, et al. Systemic exposure to rifampicin in patients with tuberculosis and advanced HIV disease during highly active antiretroviral therapy in Burkina Faso. J Antimicrob Chemother. 2012;67(2):469-72.

19. Boeree MJ, Heinrich N, Aarnoutse R, Diacon AH, Dawson R, Rehal S, et al. High-dose rifampicin, moxifloxacin, and SQ109 for treating tuberculosis: a multi-arm, multi-stage randomised controlled 
trial. Lancet Infect Dis. 2017;17(1):39-49.

20. Svensson RJ, Svensson EM, Aarnoutse RE, Diacon AH, Dawson R, Gillespie SH, et al. Greater Early Bactericidal Activity at Higher Rifampicin Doses Revealed by Modeling and Clinical Trial Simulations. J Infect Dis. 2018;218(6):991-9.

21. Byrd, Jr. RP, Mehta JB, Roy TM. Malnutrition and Pulmonary Tuberculosis. Clin Infect Dis. 2002;35(5):634-5.

\section{Tables}

Table 1: Population characteristics at baseline 
conversion

Characteristics

Total

$(n=173)$

Age (years, $\mathrm{m} \pm \mathrm{SD}$ )

Gender (Male)

Residence (Urban)

BMI $\left(\mathrm{kg} / \mathrm{m}^{2}\right)$

Years of inclusion

\begin{tabular}{|c|c|c|c|c|c|}
\hline 2016 & 13(7.51) & $9(69.23)$ & $4(30.77)$ & $9(69.23)$ & $4(30.77)$ \\
\hline 2017 & 116(67.05) & $107(92.24)$ & $9(7.76)$ & 106(91.38) & $10(8.62)$ \\
\hline 2018 & $44(25.43)$ & 40(90.91) & $4(9.09)$ & 39(88.64) & $5(11.36)$ \\
\hline HIV status (Positive) & 34(19.88) & 29(85.29) & $5(14.71)$ & $26(76.47)$ & $8(23.53)$ \\
\hline $\begin{array}{l}\text { History of TB treatment (Previously } \\
\text { treated) }\end{array}$ & $146(84.88)$ & 134(91.78) & $12(8.22)$ & 133(91.10) & 13(8.90) \\
\hline $\begin{array}{l}\text { Colony count of initial smear ( } \square 3+\text { vs }<3 \\
+)\end{array}$ & $49(28.32)$ & $43(87.76)$ & $6(12.25)$ & $43(87.76)$ & $6(12.25)$ \\
\hline $\begin{array}{l}\text { Colony count of initial culture ( } \square 3+v s<3 \\
+)\end{array}$ & $85(49.13)$ & 75 (88.24) & $10(11.76)$ & $75(88.24)$ & $10(11.76)$ \\
\hline Lung Cavities on X-ray (Yes) & 13(8.90) & 10(76.92) & $3(23.08)$ & $9(69.23)$ & $4(30.77)$ \\
\hline Cough (Yes) & 163(94.22) & $147(90.18)$ & $16(9.82)$ & 145(88.96) & 18(11.04) \\
\hline Dyspnea (Yes) & $50(28.90)$ & $39(78)$ & 11(15.28) & $38(76.00)$ & $12(24.00)$ \\
\hline Chest pain (Yes) & 72(41.62) & $61(84.72)$ & 11(15.28) & 60(83.33) & $12(16.67)$ \\
\hline Night sweat (Yes) & $91(52.60)$ & $82(90.11)$ & $9(9.89)$ & 81(89.01) & 10(10.99) \\
\hline Nausea (Yes) & $10(5.78)$ & $9(90.00)$ & $1(0.58)$ & $9(90.00)$ & $1(10.00)$ \\
\hline Vomiting (Yes) & 17(9.83) & 15(88.24) & $2(11.76)$ & 15(88.24) & 2(11.76) \\
\hline Treatment adherence (Yes) & $150(86.71)$ & $142(94.67)$ & $8(5.33)$ & 140(93.33) & $10(6.67)$ \\
\hline Leukocytes count $(\mathrm{m} \pm \mathrm{SD})$ & 8.1(3.7) & $8.0(3.8)$ & $8.9(2.0)$ & $8.1(3.8)$ & $8.0(2,8)$ \\
\hline Hemoglobin count $(m \pm S D)$ & $10.7(2.1)$ & $10.8(1.9)$ & $9.9(3.1)$ & $10.8(1.9)$ & $10.0(3.2)$ \\
\hline
\end{tabular}

\section{Converted Non-converted Converted Non-converted}

\begin{tabular}{|ccccc}
\hline $34.0(11.3)$ & $40.3(16.7)$ & $33.3(10.4)$ & $40.6(17.0)$ & $33.2(10.1)$ \\
\hline $124(71,68)$ & $114(91.94)$ & $10(8.06)$ & $114(91.94)$ & $10(8.06)$ \\
$133(76.88)$ & $119(89.47)$ & $14(10.53)$ & $117(87.97)$ & $16(12.03)$ \\
$17.8(2.8)$ & $18.0(2.8)$ & $16.4(2.4)$ & $18.0(2.8)$ & $16.4(2.3)$
\end{tabular}




\begin{tabular}{llllll} 
Platelets count $(\mathrm{m} \pm \mathrm{SD})$ & $390.2(162.9)$ & $388.5(136.2)$ & $405.0(164.7)$ & $393.5(162.3)$ & $364.8(169.8)$ \\
\hline Lymphocytes count $(\mathrm{m} \pm \mathrm{SD})$ & $1.8(1.0)$ & $1.8(0.9)$ & $1.9(1.7)$ & $1.8(0.9)$ & $2.0(1.6)$ \\
\hline Creatinine count $(\mathrm{m} \pm \mathrm{SD})$ & $78.5(19.6)$ & $78.3(19.9)$ & $79.6(17.2)$ & $78.5(19.9)$ & $78.1(17.4)$ \\
\hline Live SGOT count $(\mathrm{m} \pm \mathrm{SD})$ & $29.3(5.3)$ & $29.0(5.2)$ & $32.4(5.4)$ & $29.1(4.8)$ & $30.8(8.1)$ \\
\hline Liver SGPT count $(\mathrm{m} \pm \mathrm{SD})$ & $32.0(4.9)$ & $32.0(4.9)$ & $33.3(4.6)$ & $31.9(4.7)$ & $34.1(5.5)$ \\
\hline
\end{tabular}

$\mathrm{m}=$ mean; $\mathrm{SD}=$ standard deviation; for the categorical variable, data were expressed as $\mathrm{n}=$ number; $\%=$ percentage.

Table 2: Predictors of sputum smear and culture conversions in patients with MDR-TB, multivariate Cox proportional hazards regression analysis.

\begin{tabular}{lllll}
\hline & Estimate (SE) & $\mathrm{HR}(95 \% \mathrm{Cl})$ & p value & $\begin{array}{c}\text { Intemal validation } \\
\text { BHR, 95\% BCl }\end{array}$ \\
\hline Sputum smear conversion & & & & \\
Successful treatment (Yes vs No) & $0.471(0.248)$ & $1.602(0.984-2.605)$ & 0.05772 & $1.493(0.934-2.388)$ \\
\hline BMl $\left(\mathrm{kg} / \mathrm{m}^{2}\right)$ & $0.098(0.035)$ & $1.103(1.030-1.181)$ & $\mathbf{0 . 0 0 4 7 6}$ & $1.108(1.037-1.184)$ \\
\hline Culture conversion & & & & \\
\hline Successful treatment (Yes vs No) & $0.858(0.261)$ & $2.357(1.414-3.920)$ & $\mathbf{0 . 0 0 1 0 0}$ & $2.072(1.230-3.503)$ \\
\hline Colony count of initial culture (<3+ vs >=3+) & $0.422(0.173)$ & $1.525(1.086-2.142)$ & $\mathbf{0 . 0 1 4 8 1}$ & $1.599(1.141-2.243)$ \\
\hline History of TB treatment (Previously vs Newly) & $0.743(0.262)$ & $2.102(1.258-3.513)$ & $\mathbf{0 . 0 0 4 5 6}$ & $2.420(1.476-3.982)$ \\
\hline
\end{tabular}

$\mathrm{SE}=$ standard error, $\mathrm{HR}$ = hazard ratio, $\mathrm{Cl}$ = confidence interval, $\mathrm{BHR}=$ bootstrap hazard ratio (1,000 procedures), $\mathrm{BCI}=$ bootstrap confidence interval.

\section{Figures}




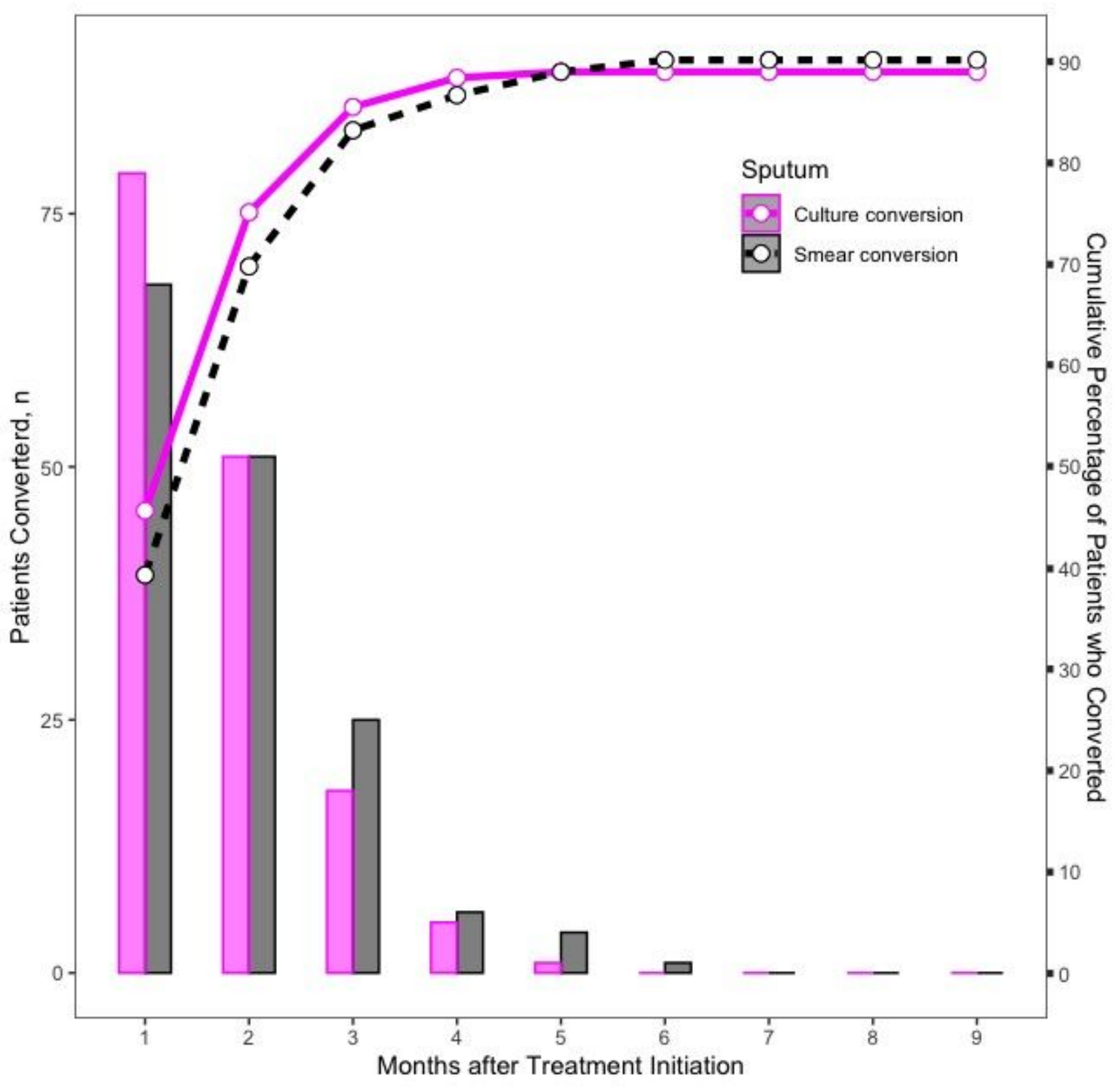

Figure 1

Initial sputum smear and culture conversion in 156 and 154 of 173 culture-positive patients who had smear and culture conversions respectively. 
A

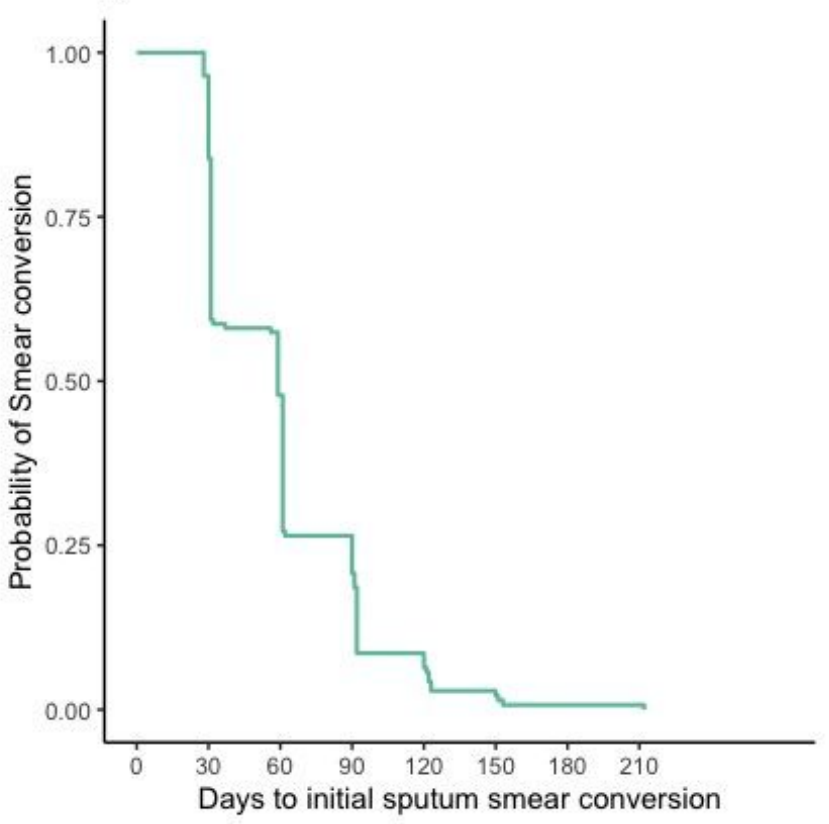

173

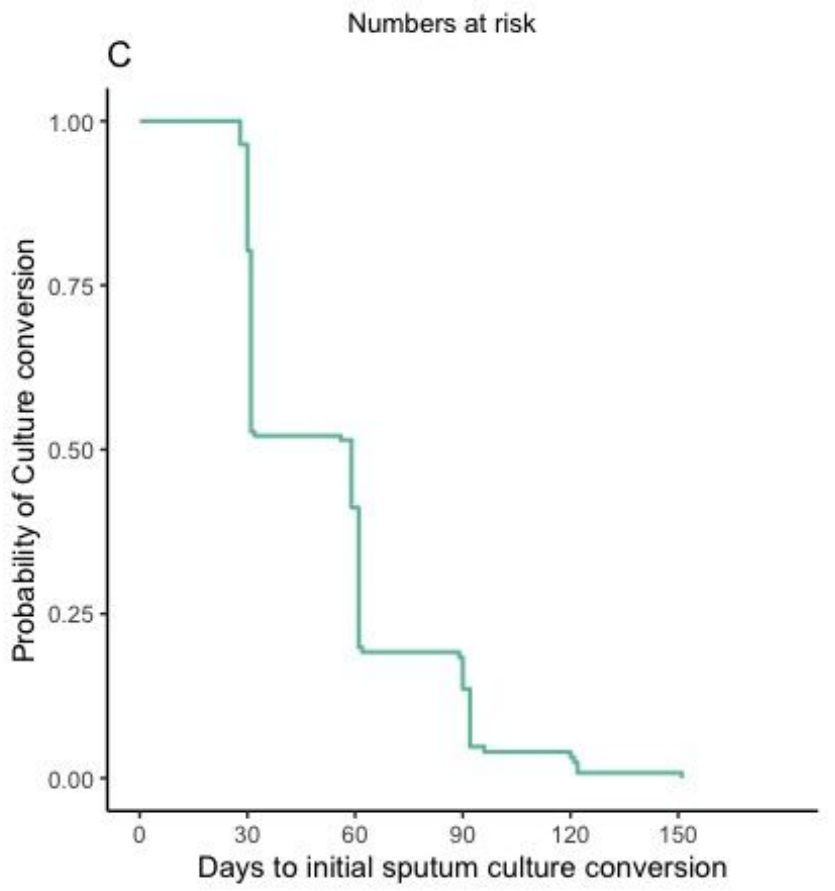

173 $\begin{array}{lllll}161 & 63 & 23 & 5 & 1\end{array}$

Numbers at risk

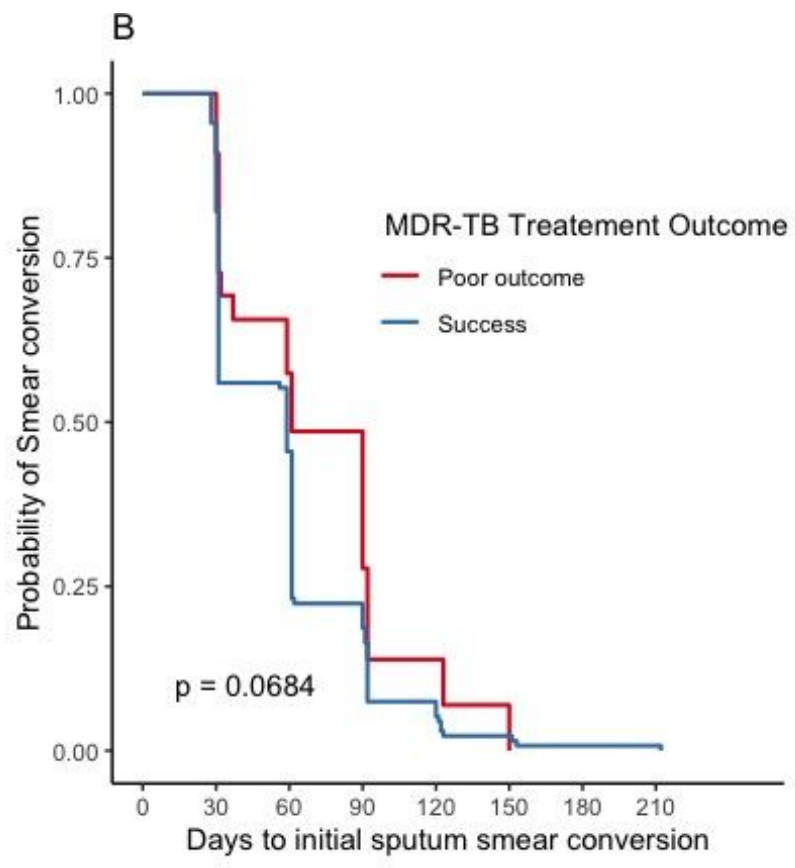

$\begin{array}{lllllllll}\text { Poor outcome } & 39 & 33 & 14 & 7 & 2 & 1 & 0 & 0\end{array}$

$\begin{array}{lllllllll}\text { Success } & 134 & 128 & 61 & 30 & 10 & 3 & 1 & 1\end{array}$

D

Numbers at risk

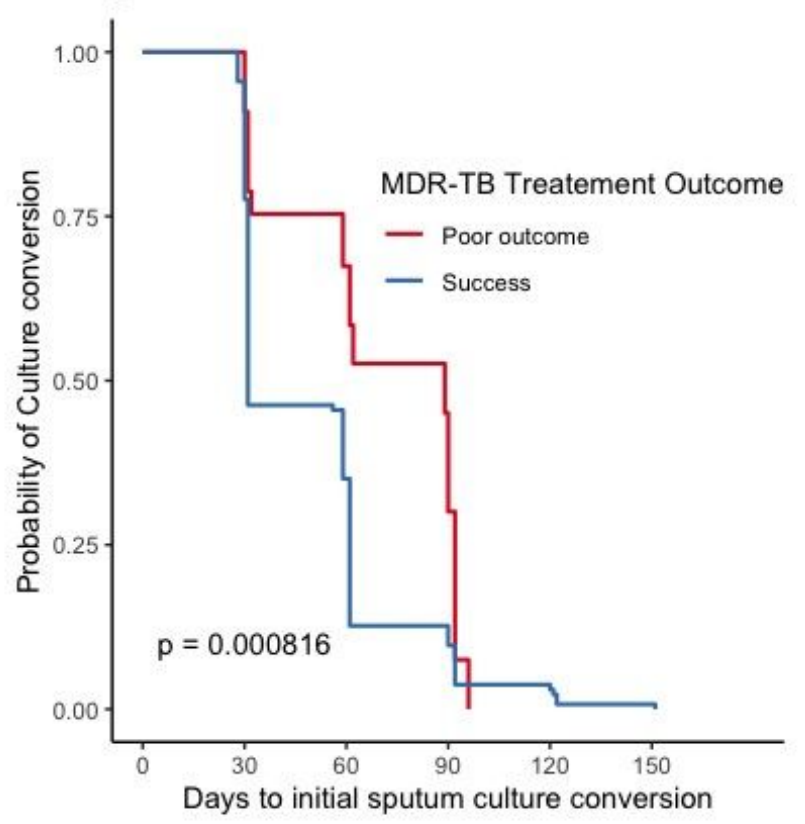

$\begin{array}{lllllll}\text { Poor outcome } & 39 & 33 & 16 & 6 & 0 & 0\end{array}$

$\begin{array}{lllllll}\text { Success } & 134 & 128 & 47 & 17 & 5 & 1\end{array}$

Numbers at risk

\section{Figure 2}

Overall time-to initial smear and culture conversions in patients with MDR-TB (A and C), and by treatment outcomes (B and D). 


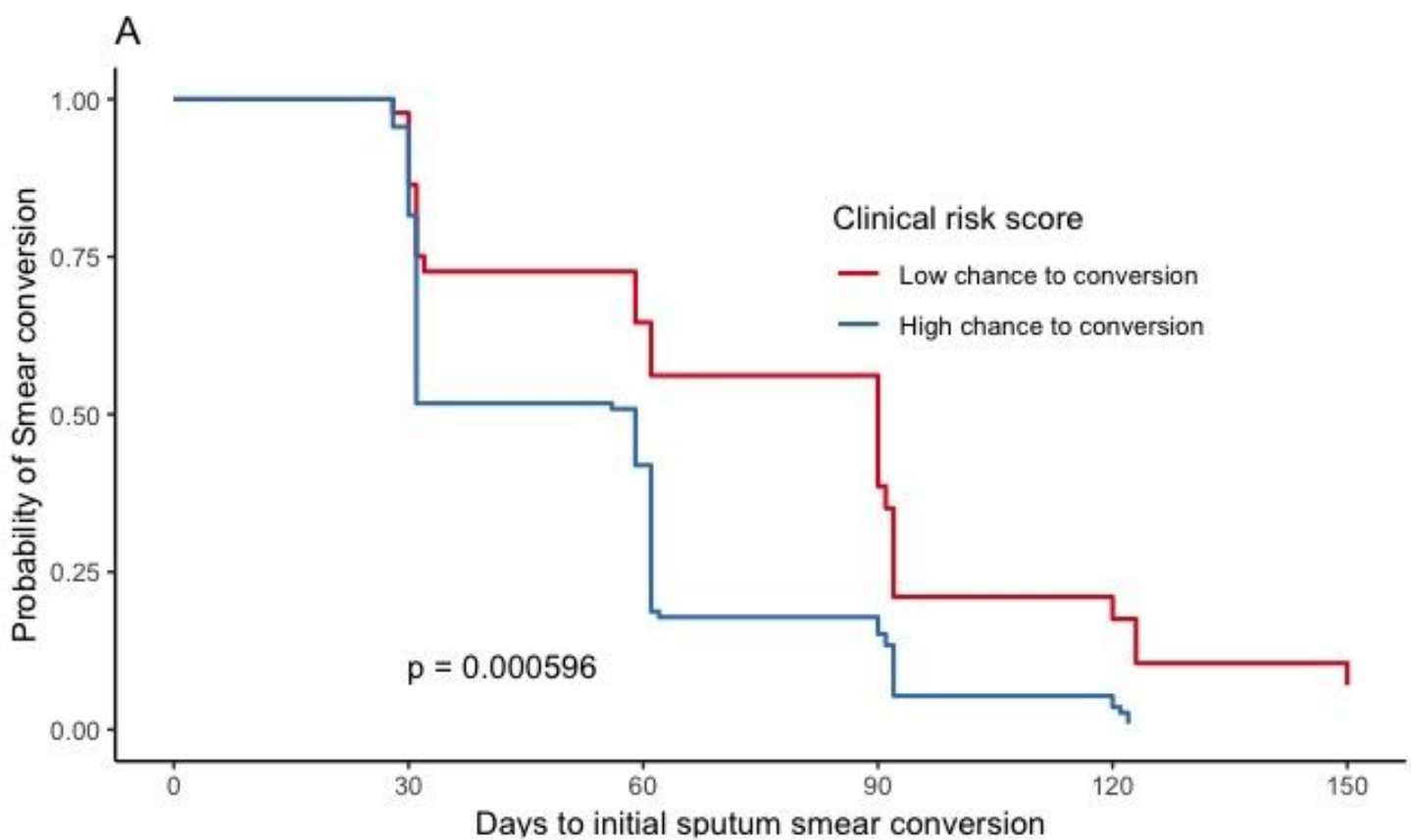

$\begin{array}{lrrrrrr}\text { Low chance to conversion } & 50 & 43 & 24 & 16 & 6 & 3 \\ \text { ligh chance to conversion } & 114 & 109 & 47 & 20 & 6 & 1\end{array}$

Numbers at risk

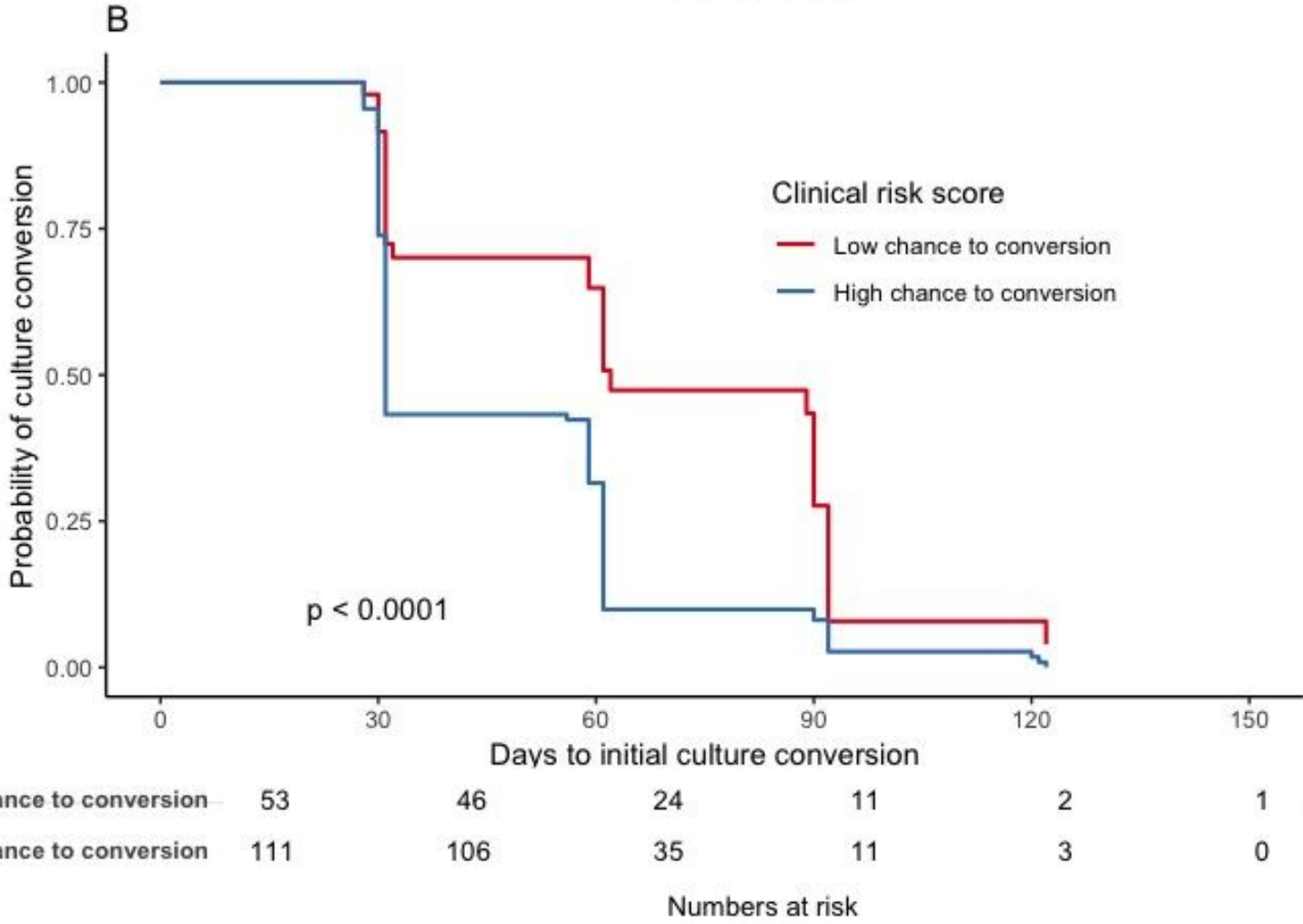

\section{Figure 3}

Time-to sputum smear and culture conversions according to the characterization groups from clinical risk score. 


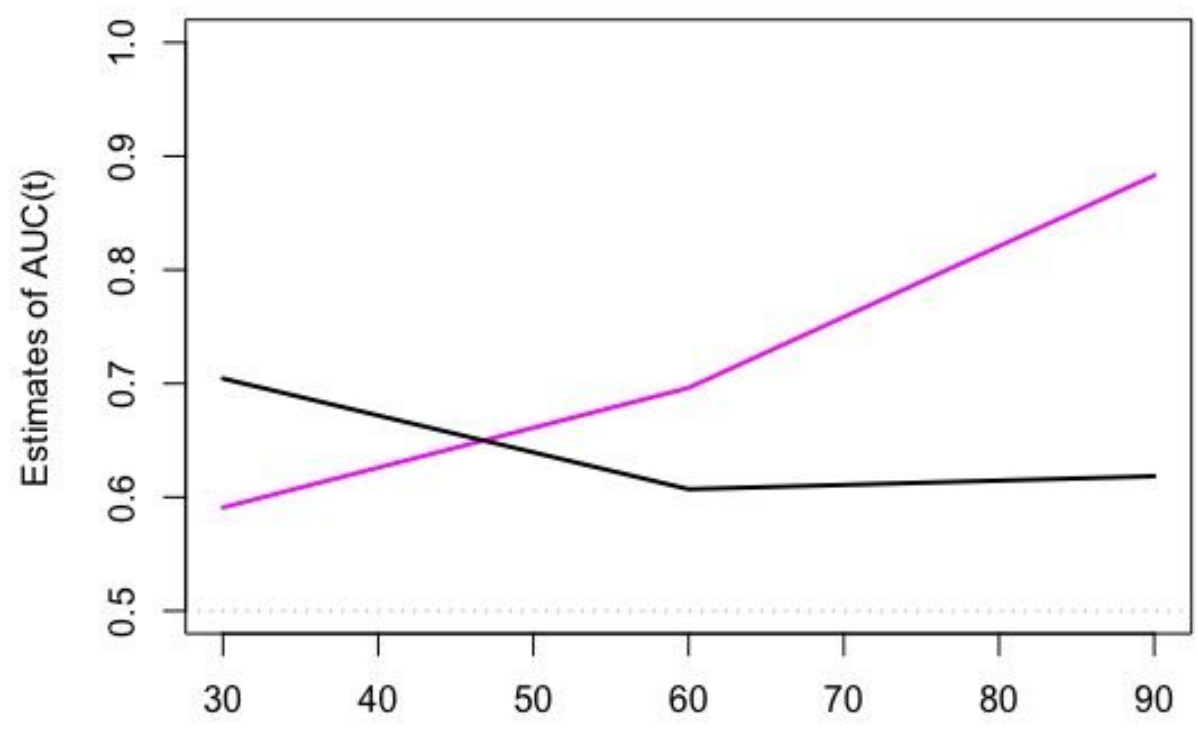

Days to initial sputum smear or culture conversion

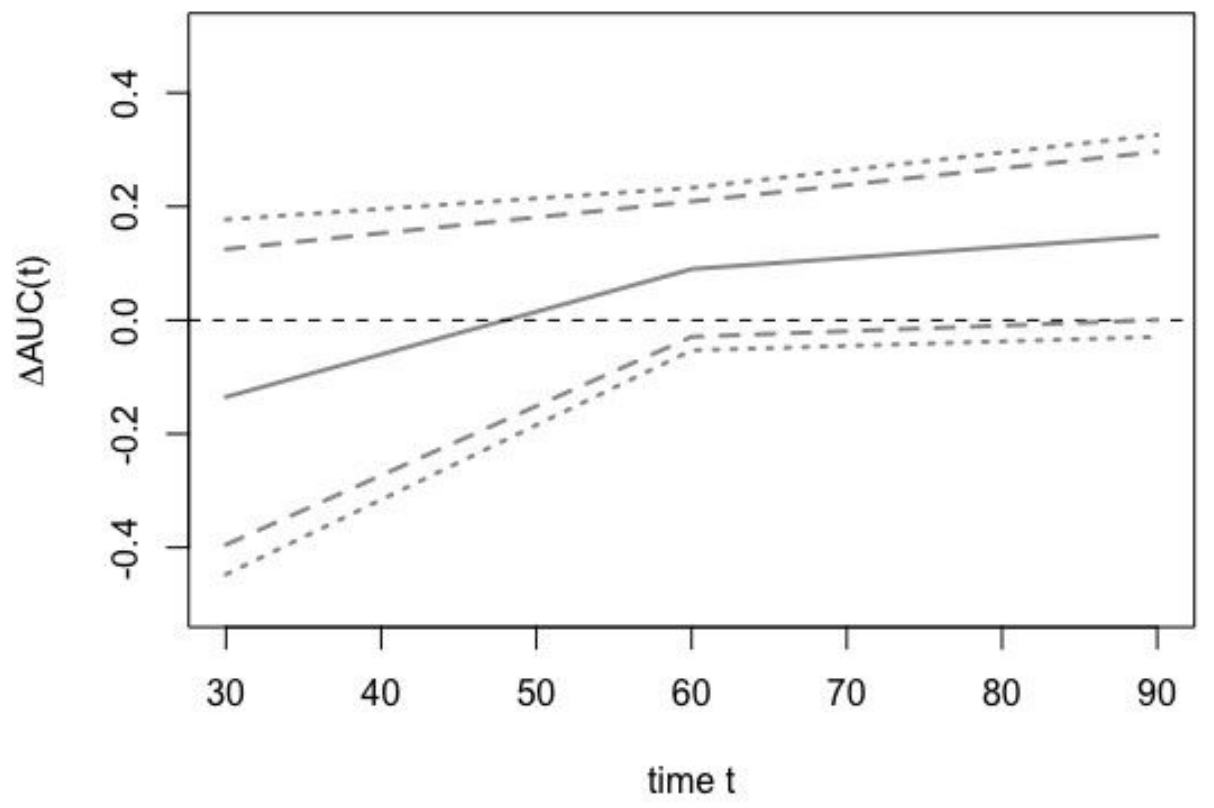

Figure 4

Comparison of the predictive accuracy of the two predicted risk of sputum smear and culture conversions:

Supplementary Files 
This is a list of supplementary files associated with this preprint. Click to download.

- sup.docx 